\title{
Comparison of ultrasound-guided interscalene block versus procedural sedation for closed shoulder reduction in a swiss university emergency department
}

\author{
Eckehart Schoell ${ }^{1 *}$, Sandra Feissli', Roland Bingisser', Stefan Ammann' ${ }^{1}$, Andreas M. Mueller ${ }^{2}$ and David L. Haeni ${ }^{2}$
}

*Correspondence: eckehart.schoell@usb.ch

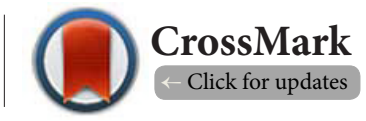

'Department of Emergency Medicine, University Hospital Basel, Basel, Switzerland.

${ }^{2}$ Department of Orthopedics and Traumatology, University Hospital Basel, Basel, Switzerland.

\begin{abstract}
Background: To facilitate shoulder reduction and shorten time from shoulder dislocation to reduction, we started in 2014 a protocol for ultrasound-guided interscalene block (UGIB) in pure shoulder dislocation as well as dislocation with greater tuberosity fracture and dislocated shoulder arthroplasty.

Methods: Within 30 month, patients with shoulder dislocation, dislocation-fracture or dislocated arthroplasty were enrolled for UGIB. Exclusion criteria were sensomotoric deficits, compromised circulation, and refusal by patient or surgeon, infection at the injection site or allergy to local anesthetics. A linear ultrasound probe was used to visualize injection of $9 \mathrm{ml}$ local anesthetics into the interscalene groove. In case of unavailability of an UGIB-trained interventionist, reduction was done under procedural sedation. Time between admission and post-reduction x-ray was registered. UGIB- and sedation-groups were compared.

Results: We encountered 168 patients (m 124, f 44) with shoulder dislocation (147), dislocation-fracture (19) or dislocated shoulder arthroplasty (2). Forty five patients (mean age 54.4y; min 17.7; max 94.8) matched UGIB-criteria; 123 patients (mean age 43.7y; min 17.0; $\max 88.1$ ) received procedural sedation. Time from admission to post reduction x-ray in the UGIB-group (MV 83min \pm 62.4 ) was significantly shorter than in the PS-group (MV 129min \pm 112.7 ). No block-related complications occurred. We got follow-up information from 42 UGIB-patients: 35 patients would opt again for UGIB, whereas 2 patients experienced the procedure as uncomfortable, 3old patients could not remember and 2 were deceased meanwhile.
\end{abstract}

Conclusions: UGIB is a reliable, fast method for shoulder reduction in shoulder dislocation, dislocationfracture and dislocated shoulder arthroplasty. Patients benefit from the shortening between shoulder dislocation and reduction.

Keywords: Shoulder dislocation, dislocated shoulder fracture, dislocated shoulder arthroplasty, shoulder reduction, interscalene nerve block, emergency department, ultrasound guided regional anesthesia

\section{Introduction}

Since antero-inferior shoulder dislocation (aSD) is the most common joint dislocation, it is a common cause for presentation at the emergency department (ED). Incidence rates (IR) in the US and Canada are estimated between 23 and 35 per $100^{\prime} 000$ person-years $[1,2]$. In 6-7 per cent aSD is combined with isolated greater tuberosity fracture [3]. Posterior dislocation (pSD) with an IR of 1.1 is a less frequent diagnosis and may often be overlooked due to occasionally minor symptoms [4]. Plenty of different reduction techniques and recommendations for muscle relaxation or procedural sedation in shoulder dislocation (SD) exist. In addition good guidance of the patient seems to be of great importance too for successful treatment [5]. Most patients with aSD, especially combined with a greater tuberosity fracture, are in a very painful condition and hardly able to relax their contracted rotator cuff. To loosen this muscular tension and avoid further compromising of the glenohumeral joint, several possibilities exist. Intravenous procedural sedation (PS) is frequently used in the ED [6]. Since these patients often have a high sympathic strain; higher doses for sedation are required 
to provide adequate muscular relaxation. This includes the risk of a loss of airway patency or hypoventilation. More recently, it has been shown that regional anesthesia (RA) in patients with SD is a further method to reduce the joint without additional sedation [7-9]. There are several advantages to this procedure. If performed by well trained staff, the method is reliable to create complete muscular relaxation of only the desired region in a conscious and pain free patient. After reduction of the shoulder and control $x$-ray, the patient can be dismissed immediately. As a result the length of stay in the ED may be reduced. In addition the patient will be pain free for several hours after discharge.

In 2014 we introduced a protocol for ultrasound guided interscalene block (UGIB) in a SD, pSD and shoulder dislocation with greater tuberosity fracture (DF) at our ED. The primary objective of our study was to create a pain free situation and to facilitate the shoulder reduction by relaxation of the rotator cuff. Secondarily, we attempted to shorten the time to reduction as well as the length of stay of the patient in our ED.

\section{Materials and methods}

After approval by the Swiss Ethics Committees on research involving humans, adult patients (age $>16$ years)with SD, DF or dislocated shoulder arthroplasty (Figure 1) who presented at our ED were enrolled for the UGIB, if a trained interventionist was available at our casualty unit at the time. Exclusion criteria for RA were sensomotoric deficits or a compromised circulation of the affected upper extremity, refusal of getting a RA by the patient or refusal of the procedure by the orthopedic surgeon, infection at the injection site or known allergy to any local anesthetics.

Patients were first seen by an emergency fellow or consultant of our ED. After precise clinical examination, standard $\mathrm{x}$-rays (antero/posterior view, y-view/outlet view) were taken to confirm suspected dislocation.

The UGIB technique we used has been described by Vincent W. S. Chan in 2003 [10]. However, for the visualization of the needle tip we only used the out-of-pane method [11]. Further, no additional nerval stimulation was applied, since the spread

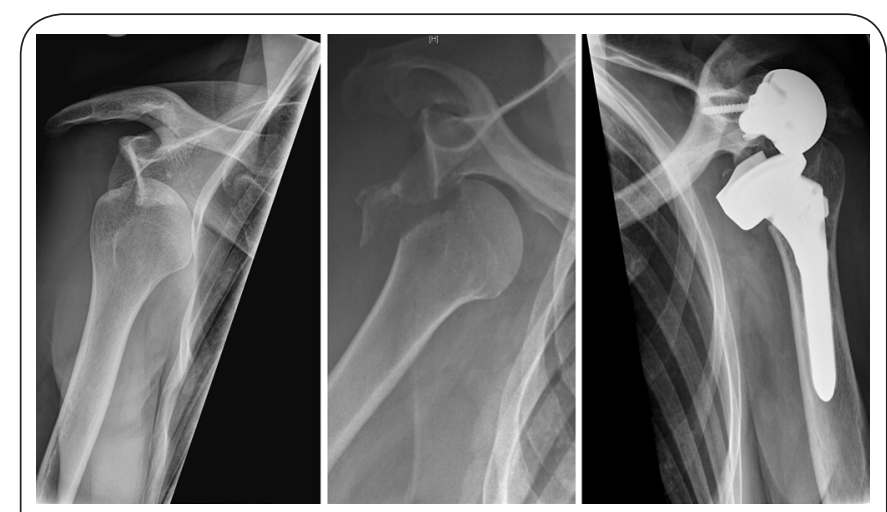

Figure 1. Pre-reduction x-ray, showing our indication for ultrasound-guided interscalene block or procedural sedation for shoulder reduction in the ED. of the local anesthetic around the brachial plexus could be observed in a real time manner (Figures 2a and 2b). Usually,
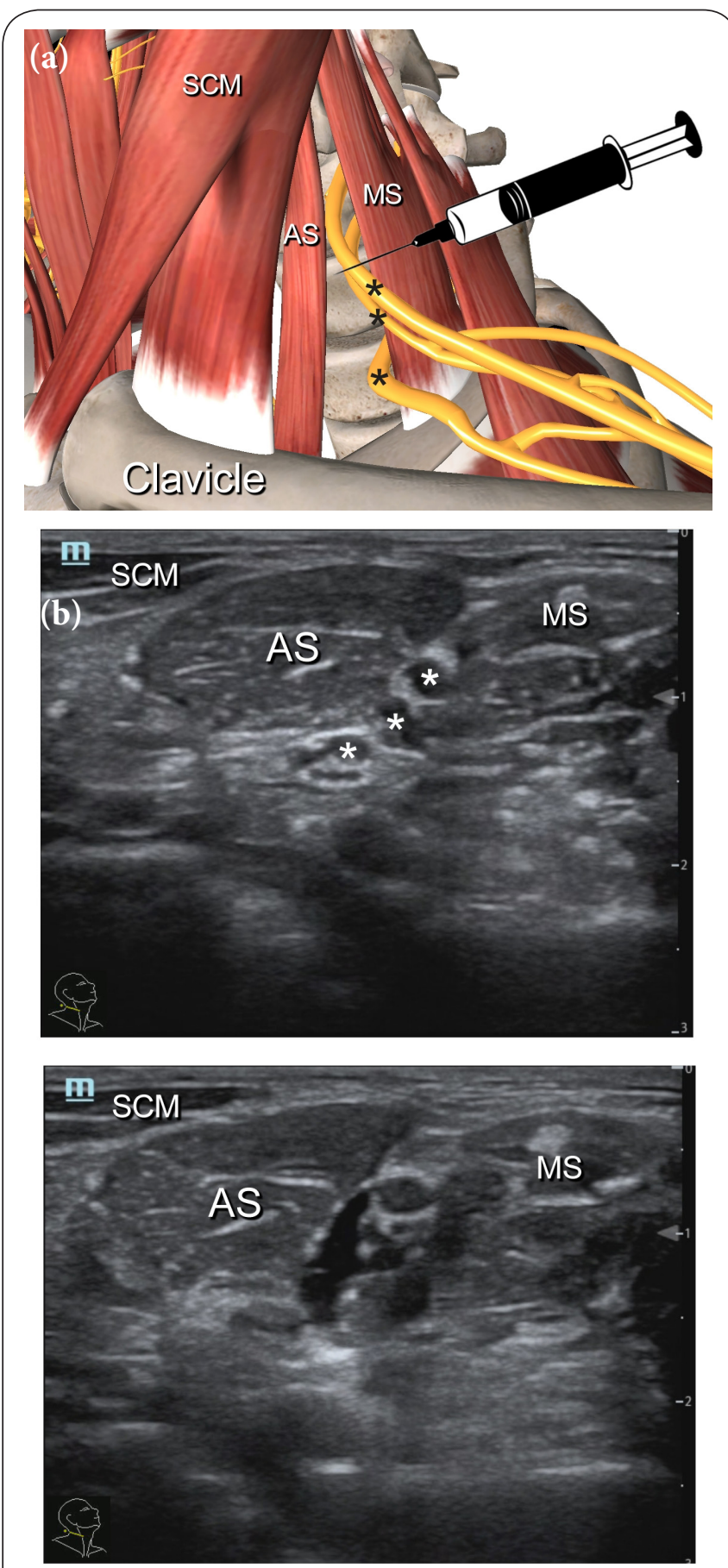

Figure 2. (a) Schema of the superior, medial and inferior trunks $\left(^{*}\right)$ of the brachial plexus lying between the anterior (AS) and medial (MS) scalene muscles, covered by the sternocleidomastoid muscle (SCM). The needle should be placed near but not through the nerves.

(b) In the sonographic view, the trunks appear as hypoechoic round structures within the interscalene groove. During the injection the spread of LA around the nerves may be visualized (figure below). 
this type of RA is used by anesthesiologists in the operation room. For this reason, a two day training course for four additional emergency physicians (EP) of our ED was organized by the first author of the study, who is an anesthesiologist and course instructor for RA. In case of unavailability of such a trained interventionist, shoulder reduction was done under PS.

For the UGIB a HITACHI Hi Vision AVIUS with a $50 \mathrm{~mm}$ linear-array probe $(5.0-13.0 \mathrm{MHz})$ or a MINDRAY TE7 with a $16 \mathrm{MHz}$ linear transducer was used. Injections were done with a PAJUNK SonoPlex Stim cannula $22 \mathrm{G} \times 50 \mathrm{~mm}$, as mentioned above without additional stimulation. Only single shot RA was applied, since extended analgesia via catheter was not intended. In DF we used $9 \mathrm{ml}$ Bupivacaine $0.5 \%$ (Sintetica SA) because of the desired prolonged duration of analgesia of at least 12 hours. In SD, as well as dislocated arthroplasty, $9 \mathrm{ml}$ Prilocaine 2\% (Sintetica SA) for shorter lasting analgesia of no more than 4 hours was utilized.

After applying RA, the reduction was done using the method described by Herkert and Ruflin in 1992 [12]. This reduction-technique is performed by a single operator, ideally in patients with smooth muscles of the rotator cuff (Figure 3 ).

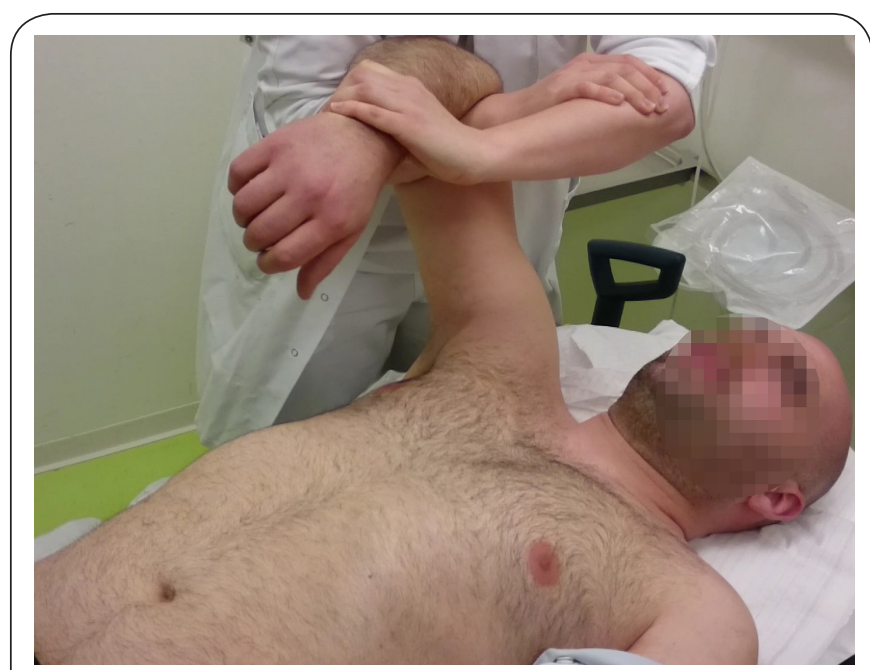

Figure 3. Shoulder reduction using the method described by Herkert and Ruflin.

X-rays to confirm adequate relocation of the humeral head were performed immediately after reduction (Figure 4). In all patients, with UGIB or PS for reduction, the time between patient's entrance to the ED and post-reduction $x$-ray was registered. In our ED, this $x$-ray should ideally bed one in an upright position, which requires the patient to be awake. Therefore, this time is a good indicator for the possibility to patients-discharge.

After a period of 30 month, the evaluation of our prospective database for ultrasound-guided interventions at our ED was performed. All patients with SD, DF or dislocated shoulder arthroplasty treated with UGIB for shoulder reduction at the ED have been compared with patients, which received PS for
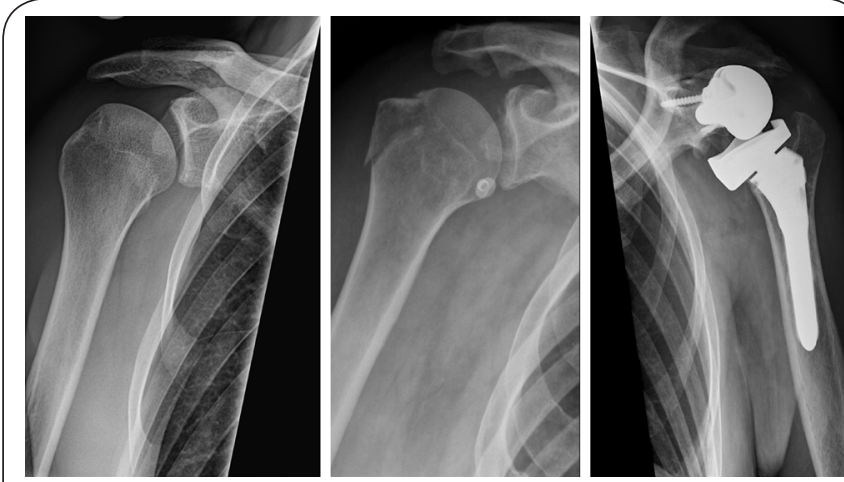

Figure 4. Post-reduction x-ray.

reduction. Since both groups are different in age and size, we used the independent $\mathrm{t}$-testing for the statistical analysis.

Moreover, we contacted the UGIB-patients by phone to obtain information about their experiences and possible complications of the UGIB.

\section{Results}

Between May 2014 and October 2016 we totally encountered 168 patients with SD, DF or dislocated shoulder arthroplasty, which were treated with closed reduction at our ED. Patient demographics are shown in Table 1. Forty five of these patients matched the inclusion criteria for UGIB whereas 123 patients received reduction under PS.

There were more patients with DF and two patients with dislocated arthroplasty in the otherwise smaller UGIB group. Since these pathologies are more frequently seen in older patients, the mean age is higher in this group (MV 54.4 years; min 17.7 years; max 94.8 years) than in the PS group (MV 43.7 years; min 17.0 years; max 88.1 years). No reduction of a shoulder arthroplasty has been done under PS within the observation period. Figure 5 characterizes the age distribution of our patients.

The time from admission to post reduction X-Ray in the UGIB group (MV $83 \mathrm{~min} \pm 62.4 \mathrm{~min}$ ) is significantly shorter than

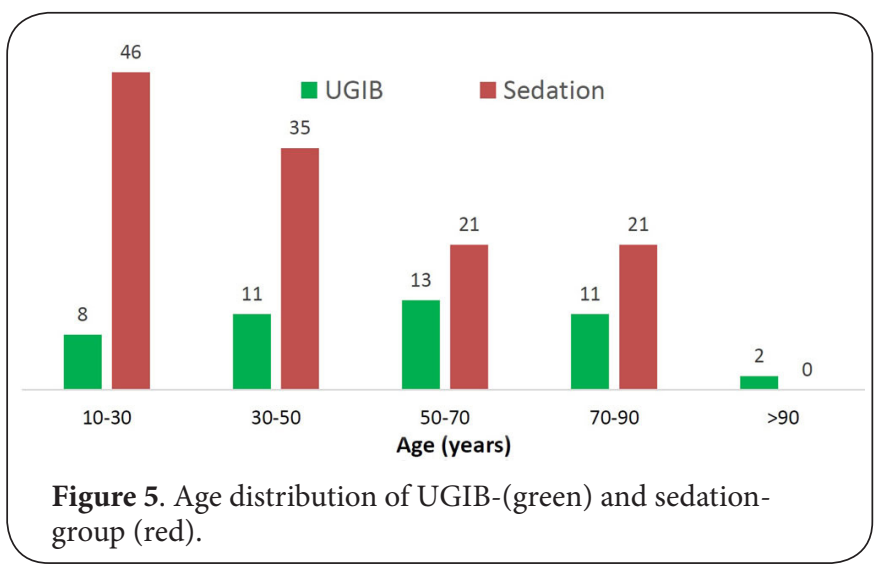


Schoell et al. Emergency Medicine \& Health Care 2017,

http://www.hoajonline.com/journals/pdf/2052-6229-5-1.pdf

doi: 10.7243/2052-6229-5-1

Table 1. Baseline demographics and time between admission and post-reduction $x$-ray.

\begin{tabular}{llllll}
\hline Type of analgesia & Type of dislocation & $\mathbf{n}$ & Gender $(\mathbf{m} / \mathbf{f})$ & Median age Yrs (IQR) & $\begin{array}{l}\text { Minutes to post reduction } \\
\text { X-ray }(\text { Median } \pm \text { StDev) }\end{array}$ \\
\hline UGIB & SD & 32 & $23 / 9$ & $53(29.4 ; 67.3)$ & $62 \pm 38.1$ \\
& DF & 11 & $5 / 6$ & $61(51.5 ; 78.3)$ & $77 \pm 69.2$ \\
& Arthroplasty & 2 & $2 / 0$ & $83.5(83.5 ; 83.5)$ & $217 \pm 77.5$ \\
\hline Procedural sedation & SD & 115 & $89 / 26$ & $34.9(26.2 ; 56.6)$ & $93 \pm 115.7$ \\
& DF & 8 & $5 / 3$ & $67.1(50.7 ; 71.4)$ & $138 \pm 50.5$ \\
\hline All UGIB & -- & 45 & $30 / 15$ & $57.4(33.6 ; 71.8)$ & $73 \pm 62.4$ \\
All Sedations & -- & 123 & $94 / 29$ & $36.8(26.9 ; 58.6)$ & $97 \pm 112.7$ \\
\hline & & & & & \\
\hline All UGIB & -- & -- & -- & -- & $83 \pm 62.4$ \\
All Sedations & -- & -- & -- & -- & $129 \pm 112.7$ \\
\hline
\end{tabular}

in the PS group (MV $129 \mathrm{~min} \pm 112.7 \mathrm{~min}$ ).

Patient files of the UGIB group have been studied to find possible block-related complications, which were defined as prolonged neurological deficit, infection at the injection site or intravascular injection. No such incident occurred. Thirty five patients have been discharged, 8 patients have been hospitalized at a surgical ward in our University hospital and two were transferred to a geriatric hospital.

Our follow-up by phone of the UGIB group revealed information from 42 of the 45 patients, 3 patients have been lost to follow-up. The question, whether they would agree to RA once again for shoulder reduction was affirmed by 35 patients, whereas two patients refused because they experienced the procedure as very uncomfortable. Three very old patients (mean age 89.25 years) could not remember and two were deceased at the meantime. Thirteen of the DF-patients (UGIB and PS) could definitively be treated in a non-operative manner, whereas four patients got an osteosynthesis of the greater tuberosity fracture later on.

\section{Discussion}

If UGIB is used instead of PS, less equipment and staff are required for patient monitoring [8]. This improves resource management in the ED allowing faster patient discharge. Unlike PS, correctly performed UGIB does not interfere with blood pressure and heart rate, an important aspect, regarding the higher age in patients with DF and shoulder arthroplasty. Standard deviation is higher in both, the UGIB and PS group, for DF and arthroplasty compared to SD. In both cases an orthopedic surgeon must be consulted before reduction, CT scan is needed for exclusion of periprosthetic fracture, resulting in prolonged waiting time until final treatment is received. Further, the general high standard deviation may be explained with considerable fluctuation in patient crowding in the ED leading to delayed post reduction $\mathrm{x}$-rays.

Patients treated with UGIB benefit from the shortening between shoulder dislocation and reduction since delays to closed shoulder reduction are associated with reduced reduction success rates in SD [13].
Compared to Blaivas et al., [8] we used the post reduction $\mathrm{x}$-ray to determine the length of stay at our $\mathrm{ED}$, since elderly patients with or without DF are often hospitalized for further treatment or social reasons. Furthermore, our study showed that dislocated reverse total shoulder arthroplasty may be reduced using UGIB too.

\section{Limitation of our study}

The UGIB and procedural sedation group differ in size and age. Due to dependency on the availability of a trained EP, no randomization was possible.

\section{Conclusions}

UGIB is a reliable, fast method for pain free shoulder reduction in SD, DF and arthroplasty in the ED, largely well accepted by the patients. EPs performing the technique must be well trained and certified, supervision of beginners is mandatory to ensure patient safety. Therefore, education in ultrasoundguided interscalene block for fellows and consultants working in emergency departments should be pursued.

\section{Competing interests}

The authors declare that they have no competing interests.

Authors' contributions

\begin{tabular}{|l|c|c|c|c|c|c|}
\hline Authors' contributions & ES & SF & RB & SA & AMM & DLH \\
\hline Research concept and design & $\checkmark$ & -- & $\checkmark$ & -- & $\checkmark$ & $\checkmark$ \\
\hline Collection and/or assembly of data & $\checkmark$ & $\checkmark$ & -- & $\checkmark$ & -- & -- \\
\hline Data analysis and interpretation & $\checkmark$ & $\checkmark$ & -- & -- & -- & -- \\
\hline Writing the article & $\checkmark$ & $\checkmark$ & -- & -- & -- & -- \\
\hline Critical revision of the article & -- & $\checkmark$ & $\checkmark$ & -- & -- & $\checkmark$ \\
\hline Final approval of article & $\checkmark$ & -- & -- & -- & -- & $\checkmark$ \\
\hline Statistical analysis & $\checkmark$ & -- & -- & -- & -- & -- \\
\hline
\end{tabular}

\section{Acknowledgement}

This work was supported by scientic funds of the University Hospital Basel.

\section{Publication history}

Editor: Bryan McIntosh, University of Bradford, UK.

Received: 03-Apr-2017 Accepted: 10-May-2017

Published: 21-May-2017 
Schoell et al. Emergency Medicine \& Health Care 2017,

http://www.hoajonline.com/journals/pdf/2052-6229-5-1.pdf

\section{References}

1. Zacchilli MA and Owens BD. Epidemiology of shoulder dislocations presenting to emergency departments in the United States. J Bone Joint Surg Am. 2010; 92:542-9. | Article | PubMed

2. Leroux T, Wasserstein D, Veillette $C$, Khoshbin A, Henry P, Chahal J, Austin $\mathrm{P}$, Mahomed $\mathrm{N}$ and Ogilvie-Harris D. Epidemiology of primary anterior shoulder dislocation requiring closed reduction in Ontario, Canada. Am J Sports Med. 2014; 42:442-50. | Article | PubMed

3. Dimakopoulos P, Panagopoulos A, Kasimatis G, Syggelos SA and Lambiris E. Anterior traumatic shoulder dislocation associated with displaced greater tuberosity fracture: the necessity of operative treatment. J Orthop Trauma. 2007; 21:104-12. | Article | PubMed

4. Robinson CM, Seah M and Akhtar MA. The epidemiology, risk of recurrence, and functional outcome after an acute traumatic posterior dislocation of the shoulder. J Bone Joint Surg Am. 2011; 93:1605-13. Article I PubMed

5. Lachance PA. Reduction of shoulder dislocation: are communication and adequate relaxation more important than technique? Can Fam Physician. 2012; 58:1189-90. | Article | PubMed Abstract | PubMed FullText

6. Dunn MJ, Mitchell R, Souza CD and Drummond G. Evaluation of propofol and remifentanil for intravenous sedation for reducing shoulder dislocations in the emergency department. Emerg Med J. 2006; 23:57-8. | Article | PubMed Abstract | PubMed FullText

7. Blaivas $M$ and Lyon M. Ultrasound-guided interscalene block for shoulder dislocation reduction in the ED. Am J Emerg Med. 2006; 24:293-6. | Article | PubMed

8. Blaivas M, Adhikari S and Lander L. A prospective comparison of procedural sedation and ultrasound-guided interscalene nerve block for shoulder reduction in the emergency department. Acad Emerg Med. 2011; 18:922-7. | Article | PubMed

9. Tezel O, Kaldirim U, Bilgic S, Deniz S, Eyi YE, Ozyurek S, Durusu M and Tezel N. A comparison of suprascapular nerve block and procedural sedation analgesia in shoulder dislocation reduction. Am J Emerg Med. 2014; 32:549-52. | Article | PubMed

10. Chan VW. Applying ultrasound imaging to interscalene brachial plexus block. Reg Anesth Pain Med. 2003; 28:340-3. | PubMed

11. Tomassetti $M$, Mangia $G$ and Bosco M. Ultrasound-Guided Interscalene Block: Out-of-Plane Versus In-Plane Needle Approach. Regional Anesthesia \& Pain Medicine. 2008; 33:e64.

12. Herkert $F$ and Ruflin $G$. [Baumann shoulder reposition]. $Z$ Unfallchir Versicherungsmed. 1992; 85:66-73. | Article | PubMed

13. Kanji A, Atkinson P, Fraser J, Lewis D and Benjamin S. Delays to initial reduction attempt are associated with higher failure rates in anterior shoulder dislocation: a retrospective analysis of factors affecting reduction failure. Emerg Med J. 2016; 33:130-3. | Article | PubMed

\section{Citation:}

Schoell E, Feissli S, Bingisser R, Ammann S, Mueller AM and Haeni DL. Comparison of ultrasound-guided interscalene block versus procedural sedation for closed shoulder reduction in a swiss university emergency department. Emerg Med Health Care. 2017; 5:1. http://dx.doi.org/10.7243/2052-6229-5-1 